\title{
Patient Characteristics Associated with Telemedicine Use at a Large Academic Health System Before and After COVID-19
}

J Gen Intern Med 36(4):1166-8

DOI: $10.1007 / \mathrm{s} 11606-020-06544-0$

(C) Society of General Internal Medicine 2021

\section{INTRODUCTION}

The COVID-19 pandemic has led to the rapid adoption of telemedicine. Health systems nationwide moved quickly to scale their telemedicine capabilities, and the Centers for Medicare \& Medicaid Services (CMS) and other payers expanded reimbursement for telehealth visits. ${ }^{1}$ While widely lauded as a means to improve value of care, telemedicine may exacerbate disparities in health care access. ${ }^{2}$ In this study, we describe the increased adoption of telemedicine at a large academic health system since the pandemic and examine our hypothesis that this increase is associated with widening racial, ethnic, and socioeconomic differences in access to care.

\section{METHODS}

Our analytic sample included all adult non-surgical and surgical ambulatory visits at the University of California, Los Angeles (UCLA) Health System that occurred between December 1, 2019, and June 30, 2020. We evaluated encounters encoded within the health record as either in-person office visits or telemedicine visits, including both video and telephone encounters. Our post-period was defined as beginning March 19, the date the California stay-at-home order was issued. ${ }^{3}$ We assessed several patient-level variables including age, sex, self-reported race and ethnicity, primary language, insurance status, and disease burden as measured by the number of listed patient comorbidities in the health record. We also estimated patient distance from clinic $^{4}$ and linked median household income to patient zip code ${ }^{5}$ using data published by the United States Census Bureau.

We first conducted unadjusted analyses quantifying characteristics of the telemedicine and in-person patient populations before and after COVID-19. We then constructed multilevel mixed effects logistic regression models for each time period, controlling for demographic and clinical covariates, to

Received October 23, 2020

Accepted December 21, 2020

Published online January 27, 2021 identify individual characteristics independently associated with telemedicine use before and after COVID-19. The models included clustering by patient ID to correct for intrapatient correlation. All hypothesis tests were two-sided and a $p$ value below 0.05 was considered statistically significant. The study was approved by the UCLA Institutional Review Board (IRB).

\section{RESULTS}

In total, 3371 out of 644,630 visits $(0.5 \%)$ were conducted via telemedicine from December 1 to March 18 compared with 186,127 out of 451,577 visits (41.2\%) from March 19 to June 30. Most telemedicine encounters were video visits (pre-pandemic $=96.0 \%$, post-pandemic $=98.2 \%$ ), with the remaining visits conducted via telephone.

Patient characteristics of telemedicine and in-person care users before and after COVID-19 are summarized in Table 1 . Multivariable regression analyses (Table 2) revealed that, both before and after COVID-19, patients aged 65 years or older, non-English speaking patients, male patients, and Medicareinsured and uninsured patients had lower adjusted odds of telemedicine use compared with patients under 65 years, English-speaking patients, female patients, and patients with commercial insurance, respectively. Additionally, after the pandemic onset, patients residing in low- and middle-income zip codes, Asian-American and multiracial patients, Latinx patients, and patients with Medicaid coverage had lower odds of having a telemedicine encounter than patients residing in high-income zip codes, White patients, non-Latinx patients, and patients with commercial insurance, respectively. The adjusted odds ratios for women and Medicare patients moved closer to 1.0 after the pandemic onset, suggesting that the predominance of female patients and underrepresentation of Medicare patients among telemedicine users lessened between time periods.

\section{DISCUSSION}

Our results show differences in telemedicine utilization by age, primary language, and insurance status that pre-date the pandemic. After the onset of the pandemic, we also observed lower rates of telemedicine use among Latinx patients, Asian- 
Table 1 Patient Characteristics by Encounter Type from December 1, 2019, to June 30, 2020

\begin{tabular}{|c|c|c|c|c|}
\hline & \multicolumn{2}{|c|}{ December 1-March 18} & \multicolumn{2}{|l|}{ March 19-June 30} \\
\hline & $\begin{array}{l}\text { In-person } \\
(n=278,986)\end{array}$ & $\begin{array}{l}\text { Telemedicine } \\
(n=2848)\end{array}$ & $\begin{array}{l}\text { In-person } \\
(n=139,903)\end{array}$ & $\begin{array}{l}\text { Telemedicine } \\
(n=105,050)\end{array}$ \\
\hline Mean age in years (SD) & $53.5(18.4)$ & $45.1(16.3)$ & $55.0(18.2)$ & $49.6(17.2)$ \\
\hline $\begin{array}{l}\text { Median distance from clinic in } \\
\text { miles (IQR) }\end{array}$ & $7.0(3.5-16.3)$ & $10.5(4.6-23.6)$ & $7.1(3.5-16.3)$ & $7.3(3.6-16.9)$ \\
\hline $\begin{array}{l}\text { Median household income* } \\
\text { (IQR) }\end{array}$ & $\begin{array}{l}\$ 110,645 \\
(\$ 82,511-\$ 130,779)\end{array}$ & $\begin{array}{l}\$ 111,094 \\
(\$ 83,482-\$ 133,136)\end{array}$ & $\begin{array}{l}\$ 108,570 \\
(\$ 78,733-\$ 130,625)\end{array}$ & $\begin{array}{l}\$ 110,645 \\
(\$ 82,511-\$ 130,876)\end{array}$ \\
\hline $\begin{array}{l}\text { Sex }(n, \%) \\
\text { Female } \\
\text { Male }\end{array}$ & $\begin{array}{l}161,237(98.9 \%) \\
117,730(99.2 \%)\end{array}$ & $\begin{array}{l}1861(1.1 \%) \\
987(0.8 \%)\end{array}$ & $\begin{array}{l}80,515(56.5 \%) \\
59,371(58.0 \%)\end{array}$ & $\begin{array}{l}62,026(43.5 \%) \\
43,011(42.0 \%)\end{array}$ \\
\hline $\begin{array}{l}\text { Race }(n, \%) \\
\text { White } \\
\text { Black } \\
\text { Asian } \\
\text { American Indian } \\
\text { Pacific Islander } \\
\text { Multiple races } \\
\text { Other/unknown }\end{array}$ & $\begin{array}{l}155,367(98.9 \%) \\
12,295(98.9 \%) \\
24,841(99.1 \%) \\
803(99.3 \%) \\
499(99.0 \%) \\
9313(98.9 \%) \\
75,868(99.1 \%)\end{array}$ & $\begin{array}{l}1661(1.1 \%) \\
135(1.1 \%) \\
233(0.9 \%) \\
6(0.7 \%) \\
5(1.0 \%) \\
99(1.1 \%) \\
709(0.9 \%)\end{array}$ & $\begin{array}{l}77,510(56.3 \%) \\
6717(56.7 \%) \\
11,436(57.9 \%) \\
395(57.6 \%) \\
224(53.0 \%) \\
4769(58.0 \%) \\
38,852(58.6 \%)\end{array}$ & $\begin{array}{l}60,200(43.7 \%) \\
5130(43.3 \%) \\
8326(42.1 \%) \\
291(42.4 \%) \\
199(47.0 \%) \\
3453(42.0 \%) \\
27,451(41.4 \%)\end{array}$ \\
\hline $\begin{array}{l}\text { Ethnicity }(n, \%) \\
\text { Latinx } \\
\text { Non-Latinx }\end{array}$ & $\begin{array}{l}32,548(98.8 \%) \\
246,438(99.0 \%)\end{array}$ & $\begin{array}{l}389(1.2 \%) \\
2459(1.0 \%)\end{array}$ & $\begin{array}{l}17,618(58.0 \%) \\
122,285(57.0 \%)\end{array}$ & $\begin{array}{l}12,755(42.0 \%) \\
92,295(43.0 \%)\end{array}$ \\
\hline $\begin{array}{l}\text { Primary language }(n, \%) \\
\text { English } \\
\text { Non-English }\end{array}$ & $\begin{array}{l}265,451(99.0 \%) \\
13,535(99.5 \%)\end{array}$ & $\begin{array}{l}2777(1.0 \%) \\
71(0.5 \%)\end{array}$ & $\begin{array}{l}132,329(56.5 \%) \\
7574(70.5 \%)\end{array}$ & $\begin{array}{l}101,884(43.5 \%) \\
3166(29.5 \%)\end{array}$ \\
\hline $\begin{array}{l}\text { Insurance status }(n, \%) \\
\text { Commercial } \\
\text { Medicare } \\
\text { Medicaid } \\
\text { UCLA Managed Care }{ }^{\dagger} \\
\text { Uninsured } \\
\text { Other/unknown } \\
\text { Mean comorbidities (SD) }\end{array}$ & $\begin{array}{l}121,816(99.0 \%) \\
64,483(99.7 \%) \\
4081(99.1 \%) \\
30,202(98.8 \%) \\
4825(99.2 \%) \\
53,579(98.3 \%) \\
8.5(8.3)\end{array}$ & $\begin{array}{l}1279(1.0 \%) \\
215(0.3 \%) \\
36(0.9 \%) \\
353(1.2 \%) \\
39(0.8 \%) \\
926(1.7 \%) \\
10.1(10.5)\end{array}$ & $\begin{array}{l}55,418(54.2 \%) \\
35,014(66.7 \%) \\
2528(59.7 \%) \\
15,703(54.4 \%) \\
2078(59.2 \%) \\
29,162(54.4 \%) \\
9.4(9.1)\end{array}$ & $\begin{array}{l}46,751(45.8 \%) \\
17,518(33.3 \%) \\
1706(40.3 \%) \\
13,168(45.6 \%) \\
1431(40.8 \%) \\
24,476(45.6 \%) \\
9.4(9.1)\end{array}$ \\
\hline
\end{tabular}

Duplicate patient encounters were excluded within each stratum

*Based on median household income linked to patient zip code

†Commercial and Medicare Advantage plans directly contracted with UCLA Medical Group

Table 2 Adjusted Correlates of Telemedicine vs. In-Person Care Utilization Before and After the COVID-19 Pandemic

\begin{tabular}{|c|c|c|c|c|}
\hline & \multicolumn{2}{|l|}{ Before COVID-19 onset } & \multicolumn{2}{|l|}{ After COVID-19 onset } \\
\hline & Adjusted OR (95\% CI) & $p$ value & Adjusted OR (95\% CI) & $p$ value \\
\hline Age $\geq 65$ years & $0.32(0.27-0.38)$ & $<0.001$ & $0.29(0.28-0.31)$ & $<0.001$ \\
\hline Distance from clinic (miles) & $1.0004(1.0002-1.0007)$ & $<0.001$ & $1.0007(1.0006-1.0008)$ & $<0.001$ \\
\hline \multicolumn{5}{|c|}{ Median household income* ( $\geq \$ 100 \mathrm{~K}$ as ref.) } \\
\hline$\$ 50-100 \mathrm{~K}$ & $0.91(0.82-1.01)$ & 0.090 & $0.92(0.89-0.94)$ & $<0.001$ \\
\hline$\$ 0-50 \mathrm{~K}$ & $0.95(0.76-1.19)$ & 0.656 & $0.81(0.76-0.87)$ & $<0.001$ \\
\hline Female sex (male as ref.) & $1.43(1.29-1.59)$ & $<0.001$ & $1.15(1.12-1.19)$ & $<0.001$ \\
\hline \multicolumn{5}{|l|}{ Race (White as ref.) } \\
\hline Black & $0.88(0.69-1.11)$ & 0.287 & $0.98(0.92-1.04)$ & 0.527 \\
\hline Asian & $0.94(0.78-1.13)$ & 0.521 & $0.92(0.87-0.97)$ & 0.001 \\
\hline Pacific Islander & $0.76(0.25-2.31)$ & 0.634 & $0.92(0.67-1.27)$ & 0.611 \\
\hline American Indian & $0.56(0.18-1.77)$ & 0.324 & $0.88(0.68-1.14)$ & 0.343 \\
\hline Multiple races & $0.86(0.65-1.13)$ & 0.273 & $0.81(0.75-0.87)$ & $<0.001$ \\
\hline Other/unknown & $0.94(0.82-1.06)$ & 0.303 & $0.82(0.80-0.85)$ & $<0.001$ \\
\hline Latinx ethnicity & $1.10(0.94-1.28)$ & 0.235 & $0.94(0.90-0.98)$ & 0.006 \\
\hline Non-English primary language & $0.53(0.38-0.72)$ & $<0.001$ & $0.47(0.43-0.50)$ & $<0.001$ \\
\hline \multicolumn{5}{|l|}{ Insurance (commercial as ref.) } \\
\hline Medicare & $0.41(0.34-0.51)$ & $<0.001$ & $0.89(0.85-0.93)$ & $<0.001$ \\
\hline Medicaid & $0.74(0.49-1.12)$ & 0.159 & $0.83(0.75-0.92)$ & $<0.001$ \\
\hline UCLA Managed Care ${ }^{\dagger}$ & $0.83(0.72-0.97)$ & 0.019 & $1.06(1.01-1.10)$ & 0.011 \\
\hline Uninsured & $0.55(0.34-0.89)$ & 0.016 & $0.61(0.55-0.68)$ & $<0.001$ \\
\hline Listed comorbidities & $1.021(1.015-1.026)$ & $<0.001$ & $1.020(1.019-1.022)$ & $<0.001$ \\
\hline
\end{tabular}

ref., reference group

*Based on median household income linked to patient zip code

+Commercial and Medicare Advantage plans directly contracted with UCLA Medical Group 
American patients, multiracial patients, patients residing in low- and middle-income zip codes, and patients with Medicaid coverage. These differences are consistent with previously described instances of the "digital divide" in the uptake of patient portals and remote monitoring for chronic conditions, which has been documented in older patients, patients belonging to racial and ethnic minorities, low-income patients, and uninsured patients. ${ }^{6}$ Our study was limited by including a single health system and a small telemedicine sample size pre-pandemic, possibly preventing identification of additional pre-existing differences. It is important to note that determining whether observed differences were unjust or unfair is beyond the scope of this study. Future studies should identify the complex causes of the observed differences, determine whether these differences persist over time, and evaluate whether these differences propagate disparities in health outcomes.

Acknowledgements: We thank Sitaram Vangala (UCLA) for guidance on statistical analyses.

Preeti Kakani, $B S^{1}$

Andrea Sorensen, $\mathrm{PhD}, M P P^{2}$

Jacob K. Quinton, MD, MPH ${ }^{2}$

Maria Han, $M D, M S^{2}$

Michael K. Ong, $M D, P h D^{2,3,4,5}$

Nirav Kamdar, MD, MPP, $M B A^{6}$

Catherine A. Sarkisian, MD, MSHS ${ }^{2,7}$

${ }^{1}$ David Geffen School of Medicine, University of California at Los Angeles,

Los Angeles, CA, USA

${ }^{2}$ Department of Medicine, University of California at Los Angeles,

Los Angeles, CA, USA

${ }^{3}$ Department of Medicine, Veterans Affairs Greater Los Angeles Healthcare System,

Los Angeles, CA, USA

${ }^{4}$ VA Center for the Study of Healthcare Innovation, Implementation and Policy (CSHIIP),

Los Angeles, CA, USA

${ }^{5}$ Department of Health Policy \& Management, UCLA Fielding School of Public Health,

Los Angeles, CA, USA

${ }^{6}$ Department of Anesthesiology and Perioperative Medicine, University of California at Los Angeles,

Los Angeles, CA, USA

${ }^{7}$ Geriatrics Research Education and Clinical Center, Veterans Affairs Greater Los Angeles Healthcare System,

Los Angeles, CA, USA
Corresponding Author: Preeti Kakani, BS; David Geffen School of Medicine, University of California at Los Angeles, Los Angeles, CA, USA (e-mail: pkakani@mednet.ucla.edu).

Funding Dr. Sarkisian received support from NIH/National Institute on Aging (NIA) Midcareer Investigator Award in Patient-Oriented Research (5K24AG047899-05). NIH/NIA UCLA Resource Center for Minority Aging Research/Center for Health Improvement of Minority Elders (P3OAG021684-16), and from NIH/NCATS UCLA Clinical \& Translational Science Institute (UL1TR001881). Dr. Ong received support from VA HSR\&D (CIN 13-417).

\section{Compliance with Ethical Standards:}

Conflicts of Interest: Dr. Kamdar holds equity and is a medical advisor for HeartCloud Inc, is a seed preferred shareholder and medical advisor for HAI Solutions LLC, and has given telemedicine lectures for Medtronic. Dr. Quinton works with UnitedHealthcare on the individual exchange team. Dr. Ong receives royalties from UpToDate for their telemedicine section.

\section{REFERENCES}

1. Centers for Medicare \& Medicaid Services (CMS). Medicare and Medicaid Programs, Basic Health Program, and Exchanges; Additional Policy and Regulatory Revisions in Response to the COVID-19 Public Health Emergency and Delay of Certain Reporting Requirements for the Skilled Nursing Facility Quality Reporting Program. Federal Register. Published online May 8, 2020.

2. Nouri S, Khoong EC, Lyles CR, Karliner L. Addressing Equity in Telemedicine for Chronic Disease Management During the Covid-19 Pandemic. NEJM Catal Innov Care Deliv. 2020;1(3).

3. Executive Department, State of California. Executive Order N-33-20. Accessed September 10, 2020. https://covid19.ca.gov/img/ExecutiveOrder-N-33-20.pdf.

4. U.S. Census Bureau. Gazetteer Files. Accessed August 24, 2020. https:// www.census.gov/geographies/reference-files/time-series/geo/gazetteerfiles.html.

5. U.S. Census Bureau. 2014-2018 American Community Survey 5-year estimates. Accessed 8 Oct 2020. https://data.census.gov/cedsci/table? q=median\%20income\&tid=ACSST5Y2018.S1901\&hidePreview=false.

6. López L, Green AR, Tan-McGrory A, King RS, Betancourt JR. Bridging the Digital Divide in Health Care: The Role of Health Information Technology in Addressing Racial and Ethnic Disparities. Joint Comm J Qual Patient Saf. 2011;37(10):437-445. https://doi.org/10.1016/S15537250(11)37055-9.

Publisher's Note: Springer Nature remains neutral with regard to jurisdictional claims in published maps and institutional affiliations. 